\title{
First records of the alien spider Pandava laminata (Thorell, 1878) (Araneae: Titanoecidae) in Poland
}

\author{
Robert RozWAŁKA ${ }^{1}$ and Paweł BIELAK-BIELECKI ${ }^{2}$ \\ ${ }^{1}$ Department of Zoology, University of Maria Curie-Sklodowska, Akademicka 19, 20-033 Lublin, Poland; \\ arachnologia@wp.pl (corresponding author) \\ ${ }^{2}$ Ractawicka 11/18, 21-040 Świdnik, Poland
}

\begin{abstract}
Pandava laminata is an exotic representative of the family Titanoecidae, which has been recently introduced into Europe. Authors discuss the occurrence of P. laminata in Poland and Europe, as well as pathways of its spreading. The observations indicate that $P$. laminata is an expansive species and it may be present in many European countries. This species spreads by ornamental plants transporting (mainly orchids), and it established several populations in greenhouses in the Netherlands and Poland.
\end{abstract}

Key words: greenhouses, occurrence, non-indigenous species, pathways of spreading

\section{INTRODUCTION}

The spider species Pandava laminata (Thorell, 1878) (Araneae: Titanoecidae) occurs in tropical regions of East Africa, Madagascar, Sri Lanka, Burma, Indonesia, Philippines, southern China, Okinawa, New Guinea and French Polynesia (Chrysanthus 1967, Lehtinen 1967, Song et al. 1999, Jäger 2008, Enriquez \& Nuñeza 2014, WSC 2015). Jäger (2008) found this species in the Zoological Garden of Cologne. In addition, P. laminata was recorded in Debrecen in Hungary (Pfliegler et al. 2012, Pfliegler 2014). Since that time, no information was reported on the occurrence of this species in Europe (Arachnologische Gesellschaft 2016, Nentwig et al. 2016, WSC 2016).

In the meantime, we have observed that the species can be found in Poland.

\section{METHODS AND MATERIAL}

Since 2009 to 2015, we searched the synanthropic araneofauna in greenhouses, flower shops and large hypermarket centres in a few cities in Poland. We repeatedly observed specimens or webs of this species, on potted plants, especially with orchids Phalaenopsis (Table 1).

\section{RESULTS}

Our research showed that $P$. laminata is a widespread spider. The findings indicate that $P$. laminata is frequent in greenhouses in Poland as well in the Netherlands (Table 1, Fig. 1).

\section{Pandava Iaminata (Thorell, 1878)}

(Figs 2-9)

Male. Total length 5.5-5.8 mm $(\mathrm{n}=3)$. Cephalothorax in the cephalic part with numerous long hairs, in the thorax part sparsely hairy. The front part of cephalothorax brownish, rear part of the yellowish-brown, chelicerae black-brown. Abdomen bushy, uniform grey-olive, only base spinnerets and spinners slightly darker (Fig. 2). Legs yellowish, profusely feathered. Hairs 


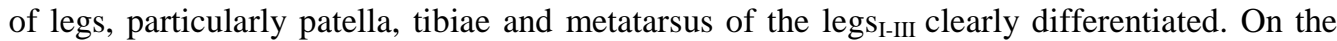
mediolateral, lateral and medioventral part of legs consisting of long, curved hairs on the side of retrodorsal and retrolateral with hair long and straight (Figs 3-4). Hair differentiation of IV

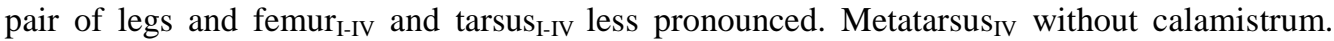
Tibia of male pedipalps with two large apophysis: semicircular retrolateral and fan-shaped, membranous, stretched on 3-4 "ribs" prolateral apophysis (Figs 7-8). Embolium thin capillaries (Figs 6-7).

Female. Total length 5.4-7.0 mm $(\mathrm{n}=3)$, colouration and body hair of the cephalothorax and abdomen close to the colouration a male, legs without differentiated hair. In the $\mathrm{Mt}_{\mathrm{IV}}$ there is long distinct calamistrum (Fig. 5). Epigyne as in Fig. 9.

Colouration of subadult specimens is similar to adults, differentiation of hair on legs absent. In juvenile and subadult specimens of both sexes calamistrum present, similar to adult females.

Remarks. Description and pictures of $P$. laminata were made shortly after undergoing preservation of specimens; after prolonged storage at significantly fade the colour of alcohol, so that the spiders look almost uniformly yellowish, yellowish-greyish coloration (see Jäger 2008: figs 8-9). Then the well reveals significantly darker, greyish (grey-black) ring at the base of spinnerets (see Jäger 2008: p. 5 and figs 8-9).

Webs of $P$. laminata are easily recognized in terms of construction, shape and type of silk, resemble the web spiders of genus Titanoeca or Amaurobius (Fig. 10). They are always placed between the protruding roots, ground and on the lower-lying shoots and leaves of plants (Fig. 10). Spiders are staying in hideouts between the roots at the base of shoots or under the leaves and they are difficult to observe and to capture.

Table 1. List of collected specimens and observation of Pandava laminata in Poland. Frequently on the labels was only general information about the country of origin (e.g. Product of Nederland (Poland); Produced in Nederland (Poland) for the commercial network market name). Collectors/Observers: P.B-B. - Paweł Bielak-Bielecki; R.R. - Robert Rozwałka; W.S. - Wojciech Starega.

\begin{tabular}{|c|c|c|c|c|c|}
\hline Site & $\begin{array}{l}\text { UTM } \\
\text { Grid }\end{array}$ & $\begin{array}{l}\text { Date of } \\
\text { collection }\end{array}$ & $\begin{array}{l}\text { Country (place) } \\
\text { of origin }\end{array}$ & Number of specimens & $\begin{array}{l}\text { Collector/ } \\
\text { Observer }\end{array}$ \\
\hline \multirow{5}{*}{$\begin{array}{l}\text { Wrocław, Długa 29/35 Str., } \\
\text { large garden-building centre } \\
\text { (on Phalaenopsis hybr.) }\end{array}$} & \multirow[t]{5}{*}{ XS 46} & 30 Mar 2010 & $\begin{array}{l}\text { The Nether-lands: } \\
\text { Bleiswijk [FT 06] }\end{array}$ & $\begin{array}{l}1 \text { juv. (grown to maturity } \\
-\delta^{\lambda} \text { ) }\end{array}$ & P.B-B. \\
\hline & & 26 Nov 2011 & $\begin{array}{l}\text { The Netherlands: } \\
\text { Bleiswijk }\end{array}$ & $1+$ (coll.), 12 exx. (obs.) & P.B-B. \\
\hline & & 24 Jan 2012 & The Netherlands & 3 exx. (obs.) & P.B-B. \\
\hline & & 28 Jan 2012 & The Netherlands & $\begin{array}{l}1 \text { exuvium }(q ?) \text { and } 15 \\
\text { exx. (obs.) }\end{array}$ & P.B-B. \\
\hline & & 26 Aug 2014 & - & 1 ex. (obs.) & P.B-B. \\
\hline $\begin{array}{l}\text { Wrocław, Długa 37/47 Str., } \\
\text { hypermarket (on Phalaenopsis } \\
\text { hybr.) }\end{array}$ & XS 46 & 26 Nov 2011 & The Netherlands & 2 exx. (obs.) & P.B-B. \\
\hline $\begin{array}{l}\text { Stężyca, Zielona } 48 \text { Str., in } \\
\text { glasshouses of large } \\
\text { ornamental plans farm (on } \\
\text { Phalaenopsis hybr.) }\end{array}$ & EC 51 & 07 Apr 2011 & Poland & $\begin{array}{l}1 \text { juv. (coll.) and } \\
\text { numerous webs obs. }\end{array}$ & R.R. \\
\hline $\begin{array}{l}\text { Lublin, Zwycięska } 1 \text { Str., large } \\
\text { garden-building centre (on }\end{array}$ & FB 07 & 24 Nov 2009 & $\begin{array}{l}\text { The Netherlands: } \\
\text { Bleiswijk }\end{array}$ & 10 webs obs. & R.R. \\
\hline Phalaenopsis hybr.) & & 23 Apr 2010 & - & 1 ex. (obs.) & P.B-B. \\
\hline & & 20 Sep 2013 & The Netherlands & 1 우 (coll.), 2 exx. (obs.) & P.B-B. \\
\hline & & 22 Nov 2012 & The Netherlands & 1 ex. (obs.) & P.B-B. \\
\hline & & $10 \mathrm{Jul} 2014$ & - & 1 ex. (obs.) & P.B-B. \\
\hline $\begin{array}{l}\text { Lublin, Orkana } 6 \text { Str., florist } \\
\text { shop (on Phalaenopsis hybr.) }\end{array}$ & FB 07 & 08 Nov 2010 & The Netherlands & $1 q$ (coll.) & P.B-B. \\
\hline
\end{tabular}


Continuation of the Table 1

\begin{tabular}{|c|c|c|c|c|c|}
\hline Site & $\begin{array}{l}\text { UTM } \\
\text { Grid }\end{array}$ & $\begin{array}{c}\text { Date of } \\
\text { collection }\end{array}$ & Country of origin & Number of specimens & $\begin{array}{l}\text { Collector/ } \\
\text { Observer }\end{array}$ \\
\hline \multirow{3}{*}{$\begin{array}{l}\text { Lublin, Turystyczna } 1 \text { Str., } \\
\text { florist shop (on Phalaenopsis } \\
\text { hybr.) }\end{array}$} & \multirow[t]{3}{*}{ FB 17} & 29 Aug 2009 & The Netherlands & $\begin{array}{l}1 \text { sub? (grown to } \\
\text { maturity) }\end{array}$ & R.R. \\
\hline & & 05 Sep 2009 & The Netherlands & 1 ex. \& two webs (obs.) & R. R. \\
\hline & & 25 Jul 2015 & - & $\begin{array}{l}1 \text { ex. (obs.) \& some webs } \\
\text { obs. }\end{array}$ & P.B-B. \\
\hline \multirow{2}{*}{$\begin{array}{l}\text { Lublin, Turystyczna } 1 \text { Str., } \\
\text { hypermarket (on Phalaenopsis } \\
\text { hybr.) }\end{array}$} & \multirow[t]{2}{*}{ FB 17} & 22 Nov 2012 & & 1 ex. (obs.) & P.B-B. \\
\hline & & 13 Jun 2013 & The Netherlands & $\begin{array}{l}1 \text { sub }+ \text { (coll.), } 4 \text { exx. } \\
\text { (obs.) }\end{array}$ & P.B-B. \\
\hline \multirow{11}{*}{$\begin{array}{l}\text { Lublin, Chemiczna } 2 \text { Str., large } \\
\text { garden-building centre (on } \\
\text { Phalaenopsis hybr.) }\end{array}$} & \multirow[t]{11}{*}{ FB 17} & 21 Nov 2009 & The Netherlands & $\begin{array}{l}1 \text { sub }{ }^{\lambda}, 1 \text { sub } q \text { (grown } \\
\text { to maturity) }\end{array}$ & R.R. \\
\hline & & 27 Nov 2009 & $\begin{array}{l}\text { The Netherlands: } \\
\text { Bleiswijk }\end{array}$ & $\begin{array}{l}1 \text { ( }(\text { coll.), and some } \\
\text { webs obs. }\end{array}$ & R.R. \\
\hline & & 15 Mar 2010 & The Netherlands & $\begin{array}{l}1 \operatorname{sub}^{\lambda}, 1 \text { sub } \\
\text { to maturity) (grown }\end{array}$ & R.R. \\
\hline & & 07 Apr 2010 & - & $\begin{array}{l}1 \text { sub }{ }^{\lambda} \text { (grown to } \\
\text { maturity) }\end{array}$ & R.R. \\
\hline & & 02 Sep 2010 & $\begin{array}{l}\text { Poland: } \\
\text { Warszawa, } \\
\text { Tomaszewski } \\
\text { LLC [DC 98] }\end{array}$ & $\begin{array}{l}1 \text { ( (coll.) \& some webs } \\
\text { obs. }\end{array}$ & R.R. \\
\hline & & 11 May 2011 & The Netherlands & 19 , and some webs obs. & R.R. \\
\hline & & $21 \mathrm{Jul} 2011$ & $\begin{array}{l}\text { The Netherlands: } \\
\text { Bleiswijk }\end{array}$ & $\begin{array}{l}1 \text { ex. (obs.) \& } 2 \text { webs } \\
\text { obs. }\end{array}$ & P.B-B. \\
\hline & & 22 Jul 2011 & $\begin{array}{l}\text { The Netherlands: } \\
\text { Bleiswijk }\end{array}$ & $\begin{array}{l}1 \text { sub }{ }^{\lambda} \text { (grown to } \\
\text { maturity) }\end{array}$ & R.R. \\
\hline & & 24 Jul 2011 & - & 6 exx. (obs.) & P.B-B. \\
\hline & & 04 Mar 2012 & The Netherlands & $10,1+$ (coll.) & R. R. \\
\hline & & $27 \mathrm{Jul} 2014$ & The Netherlands & 1 우 (coll.) & P.B-B. \\
\hline \multirow{2}{*}{$\begin{array}{l}\text { Lublin, Wolska } 11 \text { Str., } \\
\text { discount store (on } \\
\text { Phalaenopsis hybr.) }\end{array}$} & \multirow[t]{2}{*}{ FB 17} & 02 Sep 2010 & $\begin{array}{l}\text { Poland: HRS } \\
\text { Dawidy [DC 97] }\end{array}$ & $\begin{array}{l}1 \text { ( (coll.) \& some webs } \\
\text { obs. }\end{array}$ & R.R. \\
\hline & & 24 Jun 2015 & Poland & 2 webs obs. & R.R. \\
\hline $\begin{array}{l}\text { Lublin, Mełgiewska 16c Str., } \\
\text { large garden-building centre } \\
\text { (on Phalaenopsis sp. hybr.) }\end{array}$ & FB 17 & 14 Jul 2015 & The Netherlands & 3 webs. obs. & P.B-B. \\
\hline \multirow{2}{*}{$\begin{array}{l}\text { Świdnik, Krępiecka } 3 \text { Str., } \\
\text { discount sore (on Phalaenopsis } \\
\text { hybr.) }\end{array}$} & \multirow[t]{2}{*}{ FB 17} & 08 Nov 2010 & - & $\begin{array}{l}1 \text { ( (grown grown to } \\
\text { maturity) }\end{array}$ & P.B-B. \\
\hline & & 13 Nov 2010 & - & 3 exx. (obs.) & P.B-B. \\
\hline $\begin{array}{l}\text { Swidnik, Racławicka } 11 \text { Str., } \\
\text { in house }\end{array}$ & FB 17 & 15 Dec 2010 & $\begin{array}{l}\text { specimen fugitive } \\
\text { from breeding }\end{array}$ & $\begin{array}{l}1 \text { sub? (grown to } \\
\text { maturity) }\end{array}$ & P.B-B. \\
\hline \multirow{2}{*}{$\begin{array}{l}\text { Lublin, Spółdzielczości Pracy } \\
32 \text { Str., large garden-building } \\
\text { centre (on Phalaenopsis hybr.) }\end{array}$} & \multirow[t]{2}{*}{ FB 08} & 15 Feb 2013 & The Netherlands & 1 ex. (obs.) & P.B-B. \\
\hline & & 06 Aug 2015 & The Netherlands & 1 juv. (grown to maturity) & P.B-B. \\
\hline \multirow{2}{*}{$\begin{array}{l}\text { Dębówka 17a, floristic market } \\
\text { and warehouse (on } \\
\text { Phalaenopsis hybr.) }\end{array}$} & \multirow[t]{2}{*}{ FB 08} & 07 Aug 2010 & The Netherlands & 1 juv. (coll.) & R.R. \\
\hline & & 29 Aug 2010 & . & $\begin{array}{l}2 \text { exuvia (coll.) and some } \\
\text { webs obs. }\end{array}$ & R.R. \\
\hline $\begin{array}{l}\text { Elizówka 65, floristic } \\
\text { warehouse (on Phalaenopsis } \\
\text { hybr.) }\end{array}$ & FB 18 & 23 Jun 2015 & - & 2 webs (obs.) & R.R. \\
\hline \multirow{3}{*}{$\begin{array}{l}\text { Warszawa, Jana Pawła II } 82 \\
\text { Str., large garden-building } \\
\text { centre (on Phalaenopsis hybr.) }\end{array}$} & \multirow[t]{3}{*}{ DC 98} & 22 Oct 2011 & The Netherlands & 1 web (obs.) & $\begin{array}{l}\text { R.R. \& } \\
\text { W.S. }\end{array}$ \\
\hline & & 21 May 2014 & - & $\begin{array}{l}3 \text { exx. (obs.) \& some } \\
\text { webs obs. }\end{array}$ & P.B-B. \\
\hline & & 15 May 2015 & - & 2 webs (obs.) & R.R. \\
\hline $\begin{array}{l}\text { Warszawa, Radzymińska } 166 \\
\text { Str., large garden-building } \\
\text { centre (on Phalaenopsis hybr.) }\end{array}$ & EC 09 & 26 Sep 2010 & The Netherlands & 2 webs (obs.) & R.R. \\
\hline
\end{tabular}




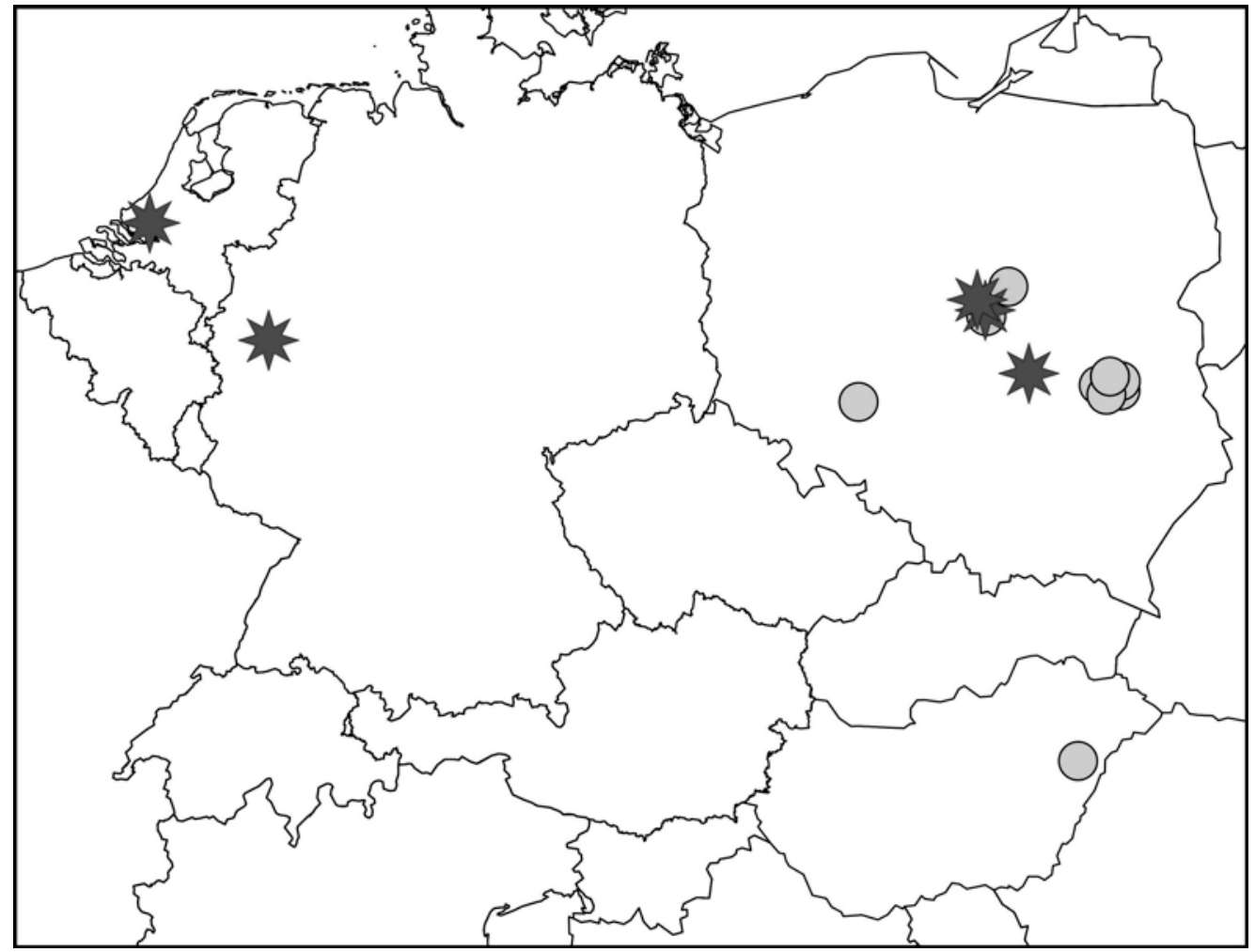

Fig. 1. Records of Pandava laminata in Europe: stars - locations of a large (stable?) population, circles - locations where single specimens were collected or observed.

\section{DISCUSSION}

Jäger (2008) revealed that the population of Pandava laminata was in the Zoological Garden of Cologne, while Pfliegler et al. (2012) found only a single specimen in Debrecen. Since then, there was no other data about $P$. laminata from Europe (Arachnologische Gesellschaft 2016, Nentwig et al. 2016, WSC 2016). Our research of the Polish synanthropic spider fauna indicate that Pandava laminata is a relatively common species (Table 1).

Studies were carried out in potential sites of occurrence: in botanical gardens of Warszawa, Kraków, Lublin and in zoological gardens of Warszawa, Wrocław and Zamość - the species was not found (R. Rozwałka, unpub. data). However, we discovered a large population of Pandava laminata in ornamental plants farm in Stężyca (Table 1, Fig. 1). In addition, based on manufacturer's data, we found two other locations inhabited by P. laminata in Poland (Table 1, Fig. 1). Besides, according to the data on the labels of the pots $P$. laminata occurs also in the Netherlands; in Poland, the most plants with $P$. laminata come from there. Thus the Netherlands is another country in Europe where the spider occurs (Table 1), although it has not been published yet (van Helsdingen 2015).

The number of records of Pandava laminata, which was found on plants imported from the Netherlands, suggests that this species is widely spread in Europe. Our observations confirm the fact that the import of ornamental plants is a major source of introduced new spider species to Europe (Kobelt 2008, Nentwig 2015). 

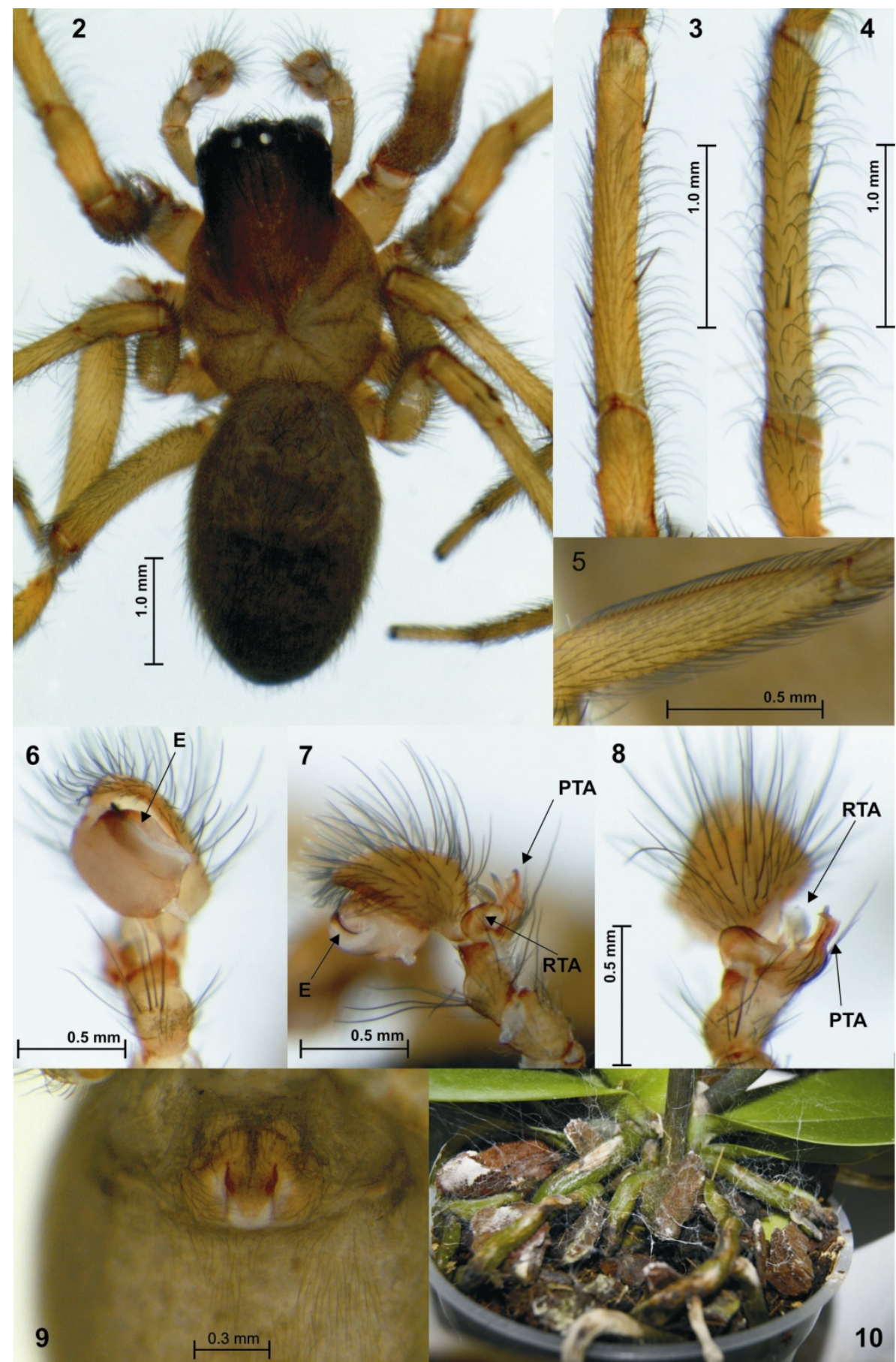

Figs 2-10. Pandava laminata (Thorell): 2 - male total view, 3 - male tibia I dorsal, 4 - male tibia I mediolateral, 5 calamistrum of metatarsus IV (female), 6 - male palp ventral, 7 - male palp lateral, 8 - male palp dorsal, 9 - female epigyne, 10 - web of Pandava laminata at Phalaenopsis hybr; PTA - prolateral tibial apophysis; RTA - retrolateral tibial apophysis; E - embolus. 


\section{REFERENCES}

Arachnologische Gesellschaft 2016. Atlas of the European Arachnids. Version 1.6. Available at http://atlas.arages.de (10 Oct 2016).

ChrYsanthus P. 1967. Spiders from south New Guinea IX. Tijdschrift voor Entomologie 110: 89-105.

ENRIQUEZ C.M.D. \& NuÑEZA O.M. 2014. Cave spiders in Mindanao, Philippines. ELBA Bioflux 6: 46-55.

JÄGER P. 2008. Pandava laminata, eine weitere nach Deutschland importierte Spinnenart (Araneae: Titanoecidae). Arachnologische Mitteilungen 36: 4-8.

Kobelt M. \& NenTwig W. 2008. Alien spider introductions to Europe supported by global trade. Diversity and Distributions 14: 273-280.

LEHTINEN P.T. 1967. Classification of the cribellate spiders and some allied families, with notes on the evolution of the suborder Araneomorpha. Annales Zoologici Fennici 4: 199-468.

NENTWIG W. 2015. Introduction, establishment rate, pathways and impact of spiders alien to Europe. Biological Invasions 17: 2757-2778. DOI 10.1007/s10530-015-0912-5

Nentwig W., Blick T., Gloor D., HÄnggi A. \& Kropf C. 2016. Spinnen Europas. Version 10.2016. Available at www.araneae.unibe.ch (10 Oct 2016).

PFLIEGLER W.P. 2014. Records of some rare and interesting spider (Araneae) species from anthropogenic habitats in Debrecen, Hungary. e-Acta Naturalia Pannonica 7: 143-156.

Pfliegler W.P., Pfeiffer K.M. \& Grabolle A. 2012. Some spiders (Araneae) new to the Hungarian fauna, including three genera and one family. Opuscula Zoologica 43: 179-186.

Song D.X., ZHU M.S. \& CHEN J. 1999. The Spiders of China. Hebei University of Science and Technology Publishing House, Shijiazhuang, 640 pp.

VAN HELSDINGEN P.J. 2015. Catalogus van de Nederlandse spinnen. Versie 2015.1. Laatst bijgewerkt: 1 mei 2015.

World Spider Catalog (WSC) 2016. Natural History Museum Bern. Version 17.5. Available at http://wsc.nmbe.ch (10 Oct 2016).

YIN C.-M. \& BAO Y.-H. 2001. Two new species of the family Titanoecidae from Hunan Province (Arachnida: Araneae). Journal of Changde Teachers University 13: 58-61.

\section{STRESZCZENIE}

\section{[Pierwsze stwierdzenie Pandava laminata (Thorell, 1878) (Araneae: Titanoecidae) w Polsce]}

W pracy omówiono występowanie $\mathrm{w}$ Polsce egzotycznego gatunku pająka $\mathrm{z}$ rodziny Titanoecidae - Pandava laminata (Thorell, 1878). Ten niedawno introdukowany gatunek znany był dotychczas w Europie jedynie z dwóch stanowisk. Zaprezentowane wyniki badań wskazują, że $P$. laminata jest pająkiem znacznie częstszym, niż wynikałoby to $\mathrm{z}$ dotychczasowych publikacji, trwale zadomowionym w Polsce i Europie. Świadczą o tym populacje tego gatunku na terenie dużych gospodarstw z roślinami ozdobnymi w Polsce. Poza szklarniami, okazy P. laminata, często obserwowano w sklepach z roślinami ozdobnymi. To właśnie transport roślin ozdobnych jest źródłem rozprzestrzeniania się tego gatunku. Ustalono także, że P. laminata występuje na terenie Holandii. 